\title{
Protective Effect of Capsaicin against Doxorubicin Induced Cardiotoxicity in Experimental Rats
}

\author{
Sanjiv Karale*, Yamuna PV, Jagadish V Kamath \\ Department of Pharmacology, Shree Devi College of Pharmacy, Mangalore, Karnataka, INDIA.
}

\begin{abstract}
Aim: The aim of the present study was to evaluate the Cardioprotective effect of Capsaicin (CAP) against Doxorubicin (DOX) induced cardiotoxicity in experimental rats. Methods: The 24 wistar rats were randomly divided into four groups (with equal numbers): Normal- 1\% Dimethyl Sulfoxide (DMSO), DOX $(15 \mathrm{mg} / \mathrm{kg}$, i.p), CAP (10 $\mathrm{mg} / \mathrm{kg}$, p.o, alone) and CAP $10 \mathrm{mg} / \mathrm{kg}$ (in combination with DOX). Cardiotoxicity was induced in wistar rats by administering DOX with a cumulative dose of $15 \mathrm{mg} / \mathrm{kg}$ (i.p) for 2 weeks. CAP with a dose of $10 \mathrm{mg} / \mathrm{kg}$ was administered in respective groups for 21 days. The influence of the treatment was analysed by quantification of serum biomarkers and antioxidants, electrocardiographic parameters and histopathological observations. Results: In the current study, the activities of Lactate Dehydrogenase (LDH), Creatine Kinase-NAC (CKNAC), Creatine Kinase-MB (CK-MB), were reduced $(P<0.001)$ in serum and Superoxide Dismutase (SOD), Reduced Glutathione (GSH) and Catalase activities were increased $(P<0.001)$ in heart tissue homogenate in all treated groups compared to DOX group. Similarly, the electrocardiographic changes were restored close to normal in all treated groups. Results were further supported by histopathological studies. Conclusion: The results reveal that CAP possesses potential benefits against cardiotoxicity induced by DOX by supressing serum markers and oxidative stress in heart tissues of experimental rats.
\end{abstract}

Key words: Cardiotoxicity, Capsaicin, Doxorubicin, Electrocardiogram, LDH, GSH.

\section{INTRODUCTION}

Doxorubicin (DOX) is an anthracycline glycoside antibiotic, derived from soil actinomycetes Streptococcus peucetius. DOX is a broad spectrum anticancer drug preferred for the treatment of solid tumors which develop in the breast, bile ducts, endometrial tissue, esophagus and liver, osteosarcomas, soft-tissue sarcomas and non-Hodgkin's lymphoma. ${ }^{1}$ The clinical usage of DOX is limited because its deleterious adverse effects like cardiotoxicity, neurotoxicity, hepatotoxicity, nephrotoxicity and testicular toxicity. The early side effects of DOX occur at a single dose like pericarditis-myocarditis syndrome, acute left-ventricular dysfunction and arrhythmias. Late effect of DOX induced cardiomyopathy is clinically manifested as congestive heart failure and exact mechanisms underlying cardiotoxicity caused by DOX are not completely elucidated. Many research studies evidenced that DOX increases inflammation and oxidative stress in the heart tissue. ${ }^{1-3}$

Various studies were demonstrated for antioxidants from the natural medicine with target to reduce oxidative stress injury evoked by DOX. Many natural antioxidants were screened to ameliorate the DOX-induced cell damage without compromising its anti-tumor efficacy in the animal studies. ${ }^{1}$ Capsaicin (trans-8-methyl-N-vanillyl-6-nonenamide) is the principal pungent phytoconstituent present in the fruits of plants fromthe genus Capsicum, belongs to family, Solanaceae. ${ }^{4}$ Beneficial uses of capsaicin range from food flavourings to therapeutics. Several literature datum of capsaicin
Submission Date: 19-05-2019; Revision Date: 17-07-2019; Accepted Date: 21-10-2019

DOI: 10.5530/ijper.54.1.11 Correspondence: Dr. Sanjiv Karale, Associate Professor, Department of Pharmacology, Shree Devi College of Pharmacy, Mangalore-574142, Karnataka, INDIA. Phone: +919591488276 E-mail: sanjiv.karale@gmail. com

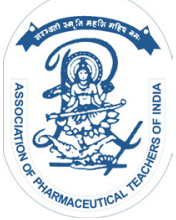

www.ijper.org 
revealed potent pharmacological effects like analgesic, antiobesity, antipruritic, anti-inflammatory, ant apoptotic, antineoplastic, free radical scavenging and neuroprotective activities. Also, recent studies showed its clinical efficacy in treatment of vascular-related disorders, metabolic syndrome and gastro protective effects. The dearth of vital medicines for prevention and management of such diseases influences the urge for further research into the pharmacological aspects of capsaicin. The present study was undertaken to evaluate the effect of capsaicin in alleviating DOX induced cardiotoxicity in experimental rats. ${ }^{5}$

\section{MATERIALS AND METHODS}

Animals: For the present study, 24 wistar rats of either sex (250-300g) were procured from animal house of Shree Devi College of Pharmacy, Mangalore. They were acclimatized to controlled laboratory conditions of temperature $\left(25 \pm 2^{\circ} \mathrm{C}\right), 30-70 \%$ humidity and $12 \mathrm{hr}$ light-dark cycles. The rats were randomized into different experimental groups and housed for each in sanitized polypropylene cages containing sterile paddy husk as bedding. They had free accessed to standard diet and water ad libitum. All the studies conducted were approved by Institutional Animal Ethical Committee, Shree Devi College of pharmacy (SDCP/IAEC/03/2018), Mangalore, Karnataka, according to prescribed guidelines of committee for the purpose of control and supervision of experiments in animals (CPCSEA), Government of India.

Chemicals: Capsaicin was procured from Yucca Enterprises, Mumbai. DOX was procured from Cipla Ltd, Verna, Goa and other chemicals used in the present study were of analytical grade and purchased from standard pharmaceutical/ chemical companies. Biochemical kits for the estimation of biomarkers/enzymes were procured from Robonik India, Pvt Ltd, Mumbai.

\section{Effect of Capsaicin on Doxorubicin induced cardiotoxicity ${ }^{6}$}

\section{Experimental design}

The present study was designed for 22 days. After 7-8 days of acclimatization, the 24 experimental rats (healthy adult Wistar rats of both sexes) were randomly divided into four equal groups in separate polypropylene cages, six animals in each the grouping of animal was done as following.

Group-I: (Normal) - Animals treated with 1\% Dimethyl Sulfoxide (DMSO) for 21 days.

Group- II: (DOX Alone) - DOX total cumulative dose of $15 \mathrm{mg} / \mathrm{kg}$ i.p for 2 weeks in six divided dosage.
Group- III: (CAP Alone) - Animals treated with CAP at a dosage of $10 \mathrm{mg} / \mathrm{kg}$ p.o for 21 days.

Group- IV: (CAP+DOX) - CAP $10 \mathrm{mg} / \mathrm{kg}$ p.o for 21 days + DOX total cumulative dose of $15 \mathrm{mg} / \mathrm{kg}$ i.p for 2 weeks in six divided dosages.

After $24 \mathrm{~h}$ after the last administration the rat was anesthetized with ketamine $(70 \mathrm{mg} / \mathrm{kg}$ i.p.) and xylazine $(10$ $\mathrm{mg} / \mathrm{kg}$ i.p.). The recordings of ECG waves were measured on the physiography. The blood was collected by retro-orbital puncture and the serum was separated by centrifugation (3000 rpm for $15 \mathrm{~min}$ ) and used for the estimation of biomarkers. Then rats were decapitated, the heart tissues were isolated, separated from their surrounding fat and connective tissue and washed with ice-cold saline quickly. Each heart was divided into two parts. Half portion was used for biochemical study and other half portion was processed for histopathological study. ${ }^{6}$

\section{Recording of electrocardiogram (ECG) in DOX induced cardiotoxicity ${ }^{6}$}

The recording of the ECG waves were measured on the physiography at the intensity of $0.5 \mathrm{mv}$ with graph speed $100 \mathrm{~mm} / \mathrm{sec}$. ECG were taken using bipolar limb lead (I, II, III) from a rat lying straight on the back with the front legs semi flexed and hind legs extended slightly. Electrodes were subcutaneously attached to the front and hind legs. The chest lead was subcutaneously attached near to the heart towards left ventricle. Leads were connected to the physiography. Electrocardiographic parameters QRS interval, QT segment, P-R interval and RR interval were measured.

\section{Determination of cardio biomarkers}

The levels of cardio biomarkers Lactate Dehydrogenase (LDH), Creatine Kinase-NAC (CKNAC) and Creatine Kinase-MB (CK-MB) from serum (supernatant) were analyzed by using commercial kits (Robonik) by using semi auto analyser (Robonik).

\section{Endogenous antioxidants assessments}

Half portion of heart of each rat was subjected to homogenization in ice-cold 10\% Trichloro acetic acid phosphate buffer and saline (0.05M, pH7.4). The heart homogenates were centrifuged for $15000 \mathrm{rpm}$ for 15 min. The obtained supernatants were used for estimations of GSH, SOD and Catalase by colorimetric method. The GSH level of hepatic tissue was analysed by using Ellmanns method. ${ }^{7}$ Catalase enzyme activity was measured by Aebi method. ${ }^{8} \mathrm{SOD}$ enzyme activity of hepatic tissue was analysed by the method described by Kakkar et al. ${ }^{9}$ 


\section{Histopathological Study}

Specimens of heart tissues of each group were fixed in 10\% buffered formalin and processed with paraffin wax. For histopathological feature examination, $5 \mu \mathrm{m}$ sections were stained with haematoxylin and eosin for the examination using light microscope. ${ }^{10}$

\section{Statistical Analysis}

The data obtained by the various parameters was statistically evaluated by one way Analysis of Variance (ANOVA) followed by Tukey-Kramer multiple comparison tests. The mean values \pm SEM were calculated for each parameter.

\section{RESULTS}

\section{Recording of electrocardiogram in DOX induced cardiotoxicity}

Electrocardiographic determination revealed a remarkably decrease $(P<0.001)$ in heart rate of DOX group compared to normal group. CAP (Alone) group and $\mathrm{CAP}+\mathrm{DOX}$ group showed rise $(P<0.001)$ in heart rate values compared to DOX group. DOX group showed increase $(P<0.001)$ in $\mathrm{RR}$ interval compared to normal group. CAP (alone) group and CAP+DOX group were produced moderately decrease $(P<0.01)$ in $\mathrm{RR}$ interval compared to DOX group. DOX group showed increase $(P<0.001)$ in QT, QRS and PR interval compared to normal group. CAP (Alone) group and $\mathrm{CAP}+\mathrm{DOX}$ group caused decrease $(P<0.001)$ in QT interval compared to DOX group (Table 1 and Figure1-3).

\section{Estimation of cardio biomarkers}

DOX group explored increase $(P<0.001)$ in CK-MB, CK-NAC and LDH level compared to normal group. $\mathrm{CAP}$ (alone) group and CAP $+\mathrm{DOX}$ group were showed moderately decrease $(P<0.001)$ in biomarkers level compared to DOX group (Table 2 and Figure 4).

\section{Estimation of endogenous antioxidants}

In the assessment of antioxidant markers decrease $(P<0.001)$ in the level of SOD, GSH and catalase in DOX group compared to normal group were observed. CAP (Alone) group and CAP+DOX group were showed elevation $(P<0.01)$ in the content of SOD, GSH and catalase compared DOX group (Table 3 and Figure 5).

\begin{tabular}{|c|c|c|c|c|c|}
\hline Treatment & $\begin{array}{l}\text { Heart Rate } \\
\text { (beats/min) }\end{array}$ & $\begin{array}{l}\text { RR Interval } \\
\text { (ms) }\end{array}$ & $\begin{array}{l}\text { QT Interval } \\
\text { (ms) }\end{array}$ & QRS Interval (ms) & $\begin{array}{l}\text { PR Interval } \\
\text { (ms) }\end{array}$ \\
\hline Normal & $181.33 \pm 6.21$ & $192.33 \pm 5.24$ & $196.83 \pm 3.6$ & $145.83 \pm 4.11$ & $81.66 \pm 4.22$ \\
\hline DOX Alone & $102.83 \pm 5.38^{* * *}$ & $270.16 \pm 5.30^{\star * *}$ & $269.33 \pm 5.57^{* * *}$ & $198.16 \pm 1.47^{* * *}$ & $177.66 \pm 4.32^{* * *}$ \\
\hline CAP Alone & $181.66 \pm 2.80$ & $180.5 \pm 5.46$ & $195.5 \pm 2.94$ & $154.83 \pm 6.55$ & $88.66 \pm 4.84$ \\
\hline$C A P+D O X$ & $163 \pm 4.73^{* * *} \# \#$ & $239.16 \pm 4.35^{\star * *} \# \#$ & $228.33 \pm 5.04^{* * *} \# \#$ & $168.16 \pm 1.47^{*} \# \#$ & $137.16 \pm 5.41^{* * *} \# \# \#$ \\
\hline
\end{tabular}

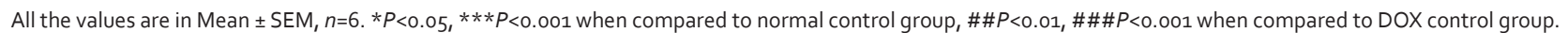

\begin{tabular}{|l|c|c|c|}
\hline \multicolumn{2}{|c|}{ Table 2: Effect of CAP on serum biomarkers in DOX induced cardiotoxicity. } \\
\hline \multirow{2}{*}{ Treatment } & \multicolumn{2}{c|}{ Serum biomarker level (U/L) } \\
\cline { 2 - 4 } & CK-MB & \multicolumn{2}{c|}{ CK-NAC } \\
\hline NORMAL & $142.33 \pm 2.25$ & $78.16 \pm 1.47$ & $263.33 \pm 3.26^{* * *}$ \\
\hline DOX Alone & $453.16 \pm 2.48^{* * *}$ & $79.33 \pm 1.96$ & $681.66 \pm 2.33^{* * *}$ \\
\hline CAP Alone & $168.5 \pm 1.76$ & $397.83 \pm 4.79$ \\
\hline CAP+DOX & $275.16 \pm 1.47^{* * \ldots \# \#}$ & $150.66 \pm 2.80^{* * \# \#}$ \\
\hline
\end{tabular}

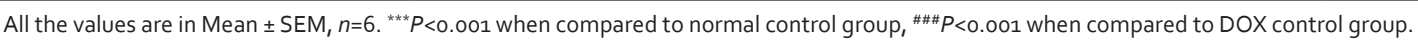

Table 3: Effect of CAP on heart tissue homogenate level of antioxidants in DOX induced cardiotoxicity.

\begin{tabular}{|c|c|c|c|}
\hline Treatment & $\begin{array}{c}\text { SOD } \\
(\mu \mathrm{g} / \mathrm{g} \text { wet gland) }\end{array}$ & $\begin{array}{c}\text { Catalase } \\
(\mu \mathrm{g} / \mathrm{g} \text { wet gland) }\end{array}$ & $\begin{array}{c}\text { GSH } \\
\text { (nM /g wet gland) }\end{array}$ \\
\hline NORMAL & $85.33 \pm 3.77$ & $55.66 \pm 5.08$ & $85.33 \pm 4.88$ \\
\hline DoX Alone & $20.5 \pm 2.16^{* * *}$ & $20.33 \pm 1.96^{* * *}$ & $36.33 \pm 4.71^{* * *}$ \\
\hline CAP Alone & $60.66 \pm 8.26$ & $83 \pm 3.16$ & $63.16 \pm 5.19$ \\
\hline CAP+DOX & $48.33 \pm 2.42^{* * * \# \#}$ & $39.33 \pm 1.36$ \#\# & $61.33 \pm 3.20 * \#$ \\
\hline
\end{tabular}

All the values are in Mean $\pm \mathrm{SEM}, n=6$. $* * P<0.01 * * * P<0.001$ when compared to normal control group, ${ }^{\# \#} P<0.01$, when compared to $\mathrm{DOX}$ control group. 


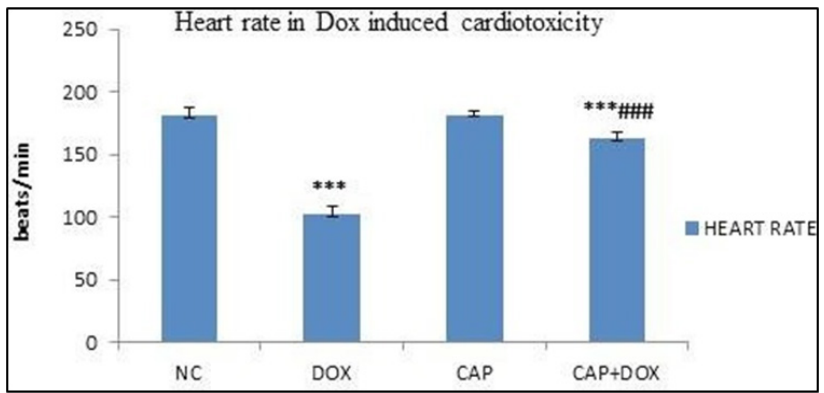

Figure 1: Effect of CAP on heart rate in DOX induced cardiotoxicity.

All the values are in Mean \pm SEM, $n=6 .{ }^{* *} P<0.001$ when compared to normal control group, \#\#\#P<0.001 when compared to DOX control group.

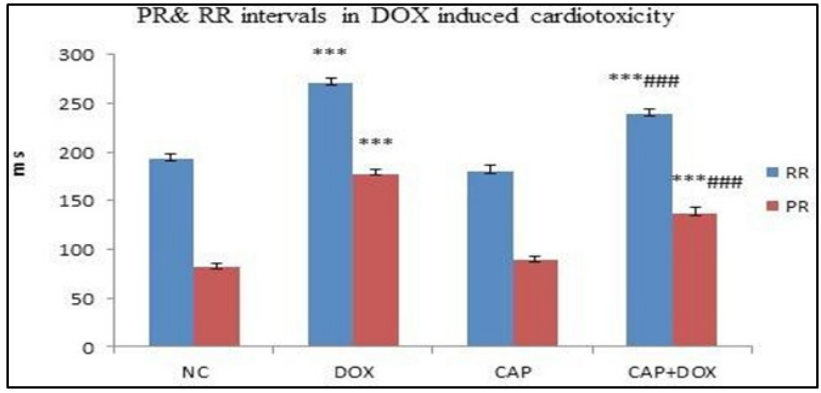

Figure 2: Effect of CAP on PR and RR intervals in DOX induced cardiotoxicity.

All the values are in Mean \pm SEM, $n=6$. ${ }^{* \star *} P<0.001$ when compared to normal control group, \#\#\#P<0.001 when compared to DOX control group.

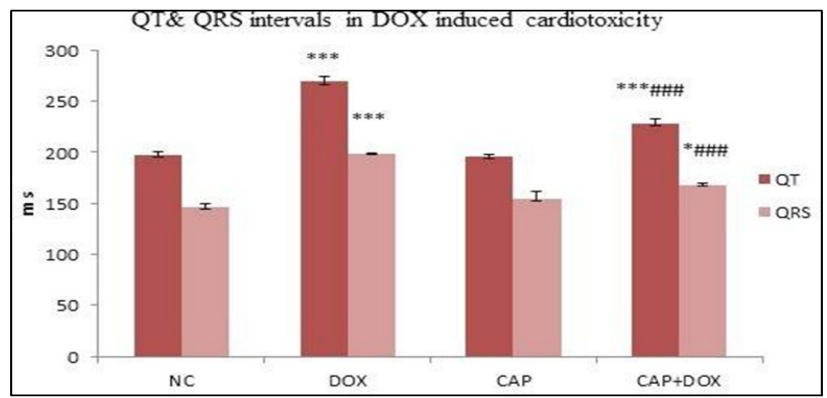

Figure 3: Effect of CAP on PR and RR intervals in DOX induced cardiotoxicity.

All the values are in Mean \pm SEM, $n=6$. ${ }^{* *} P<0.001$ when compared to normal control group, \#\# $P<0.001$ when compared to DOX control group.

\section{Histopathological investigation}

Heart tissue of normal group and CAP group showed normal texture of myocardial cell including cardiomyocytes are regular, with no visible signs of degeneration or necrosis. In between the cardiomyocytes, endomysium is in normal quantity, with no signs of fibrosis. DOX group showed vacuolization of the cardiomyocytes, infiltration of inflammatory cells, myocardial tissue separation and myofibril loss. CAP+DOX group

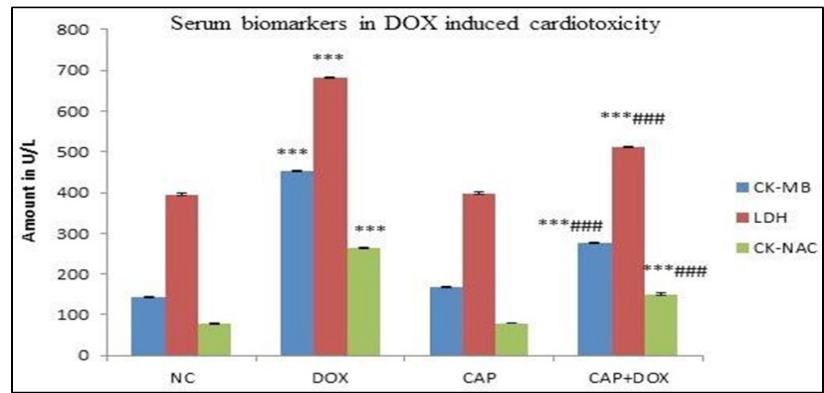

Figure 4: Effect of CAP on serum level of biomarkers in DOX induced cardiotoxicity.

All the values are in Mean $\pm S E M, n=6$. ${ }^{* *} P<0.001$ when compared to normal control group, \#\# $P<0.001$ when compared to DOX control group.

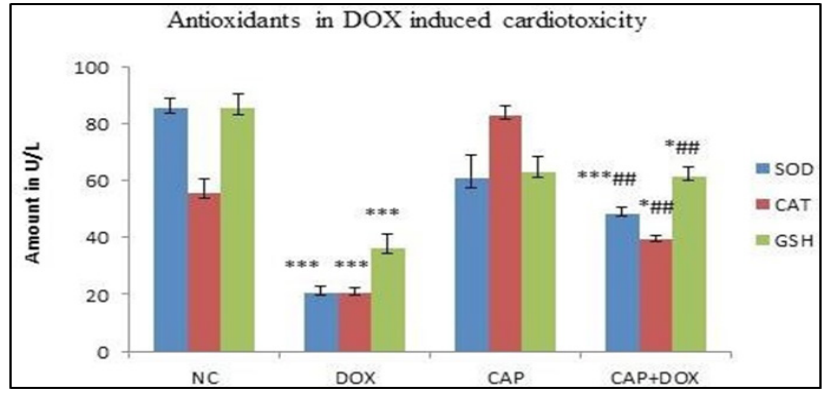

Figure 5: Effect of CAP on antioxidant level in DOX induced cardiotoxicity.

All the values are in Mean \pm SEM, $n=6 .{ }^{* *} P<0.001$ when compared to normal control group, \#\#\#P<0.001 when compared to DOX control group.
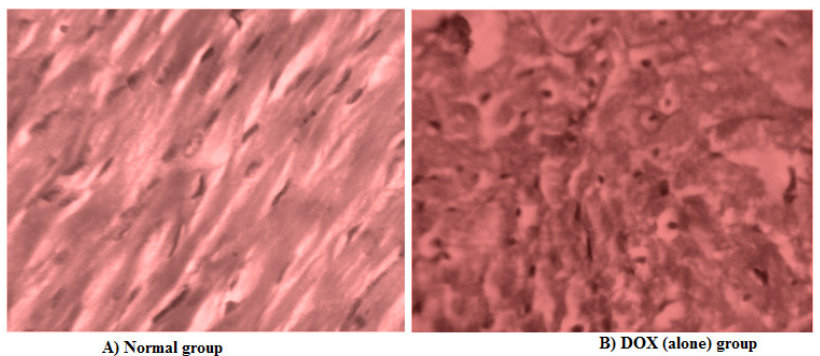

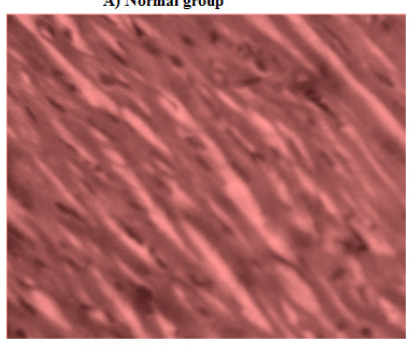

C) CAP (alone) group

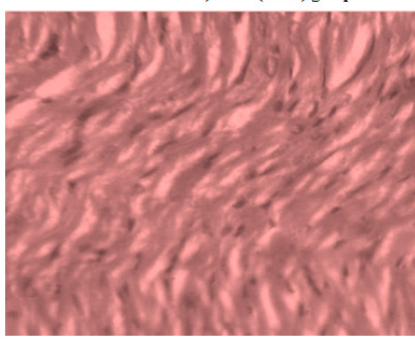

D) $\mathrm{CAP}+\mathrm{DOX}$ group
Figure 6: Histological architecture of heart tissues in different experimental groupsin DOX induced cardiotoxicity. (H and E, $X 400)$. $H$ and $E=$ Hematoxylin and Eosin

demonstrated protective efficiency of capsaicin, showed decreased infiltration of leukocytes, fewer intracellular spaces within the myocardium and lesser defragmentation of myofibrils (Figure 6). 


\section{DISCUSSION}

The current study we evaluated the potential effect of CAP on DOX induced cardiotoxicity in wistar rats. Anticancer drugs contribute significantly to the global burden of adverse drug reactions. ${ }^{11}$ DOX evoked cardiotoxicity is a major clinical challenge to its ubiquitous therapeutic use in oncology. DOX provide its potential anticancer activity towards various kinds of tumors by its cytotoxic effect which involves several interactive mechanisms like inhibition of macromolecular biosynthesis by DNA intercalation as well as its cytotoxic action is manifested by suppression of topoisomerase II. The previous literature data of DOX illustrated the role of DOX in escalating free radical synthesis which in turn leads to cytotoxicity in many vital organs such as heart, brain, liver and kidney. ${ }^{12,13}$

CAP is an alkaloid derived from the fruits of Capsicum frutescens, genus belonging to family Solanaceae and acts by activating the transient receptor potential vanilloid 1(TRPV1) channel. CAP possesses strong antioxidant property. CAP plays a major role in protection of lipids, proteins and enzymes from oxidative damage. The other activities observed for CAP are anti-inflammatory, antidiabetic, anti obesity, thermogenesis and anticancer, analgesic, prevention of adipogenesis, alleviate insulin resistance, ameliorate vascular dysfunction and control glucose homeostasis. ${ }^{14,15}$

Electrograph abnormalities are the main criteria generally used for the definite diagnosis of myocardial injury. An altered membrane function due to DOX induced lipid peroxidation is held responsible for most ECG changes. The consecutive loss of cellular membrane damage due to oxidative stress may be characterized by ST-elevation, T-wave become abnormally tall. ${ }^{16}$ In our study, significant alterations of ECG patterns in DOX administered rats were observed compared to normal rats. The characteristic findings were prolongation of QT interval, ST interval and widening of QRS complex. QRS intervals are directly related to cell depolarization, the QT interval is an expression of the late repolarisation phase. CAP pre-treatment showed a protective effect against DOX induced altered ECG parameter and eliminated acute fatal complications by protecting the cell membrane damage. ${ }^{17,18}$

DOX may cause deficiency of oxygen supply or glucose to the myocardial cell membrane leading to damage or rupture of cell membranes so that the enzymes leaks out. ${ }^{19}$ Any process that disrupts the sarcolemma membranes (e.g. myocarditis, cardiac trauma or cardiac surgery including endomyocardial biopsy) can release cytosolic CK-MB. In the current work, animals treated with DOX were showed a significant elevation in the levels CK-MB, CK-NAC and LDH. The DOX admin- istration causes the rapid swelling of sub-sarcolemma bulbs and injured myocardium this might be the possible mechanism for increased levels of these enzymes in serum. These findings illustrate myocardial injury and cellular disruption. ${ }^{17}$ Pre-treatment with CAP exhibits a significant decline in CK-MB, CK-NAC and LDH in DOXtreated rats. The decline in levels of serum biomarker findings in CAP administered animals might be due to its free radical scavenging action that reduces leakage of enzymes into bloodstream and stabilizes membrane permeability. ${ }^{20}$

In present study, the cardiomyopathy is caused due to generation of oxygen derived free radicals. They cause direct injury to cell membranes, which disrupts cardiac membranes. ${ }^{19}$ Oxidative stress and mitochondrial dysfunction are associated with disease and toxic process. It results from over production of reactive oxygen species (ROS). Antioxidant such as SOD, GSH and Catalase plays a crucial role in both scavenging reactive oxygen species and the detoxification of drugs. DOX administration causes a reduction in antioxidant level and generates free radicals in heart and decreases its ability to detoxify ROS. ${ }^{18,20}$ CAP pre-treatment provides a significant increase in antioxidant levels due to protective effect against oxidative stress injury induced by DOX.

In the current work, histopathological report suggests DOX administration causes various changes in the heart tissue including disruption and loss of myofibrils and vacuolization of the cytoplasm, enlarged swollen mitochondria, patchy necrosis and inflammatory cells where observed. The gross anatomical changes of the heart treated with DOX showed a typical chronic response including ventricular dilatation, inflammatory cell and overall enlargement. ${ }^{17}$ The rats pre-treated with CAP showed significant histopathological changes compared to DOX treated group. The beneficial alterations are due to the free radical scavenging property of capsaicin. ${ }^{20}$

\section{CONCLUSION}

The present study implicate that DOX administration leads to cardiac damage through cardiac membrane disruption and oxidative stress evidenced by changes in ECG alterations, cardiobiomarkers, antioxidant markers (GSH, catalase and SOD) and histopathological modulations. The pre-treatment with oral administration with CAP demonstrates protective role by its antioxidant and free radical scavenging actions in DOX -induced cardiotoxicity in rats.

\section{ACKNOWLEDGEMENT}

The authors are thankful to the Principal and Management of Shree Devi College of Pharmacy, Mangalore, 
for providing all facilities and their immense support for our work.

\section{CONFLICT OF INTEREST}

The authors declare no conflicts of interests.

\section{ABBREVIATIONS}

CAP: Capsaicin; DOX: Doxorubicin; CPCSEA: Committee for the Purpose of Control on Experiments in Animals; DMSO: Dimethyl sulfoxide; IAEC: Institutional Animal Ethics Committee; ECG: Electrocardiogram; IU: International Units; LDH: Lactate Dehydrogenase; SOD: Superoxide Dismutase; CK-NAC: Creatine Kinase-NAC, CH-MB: Creatine Kinase-MB; GSH: Reduced Glutathione; ANOVA: Analysis of variance; ROS: Reactive oxygen species; TRPV: transient receptor potential vanilloid.

\section{REFERENCES}

1. Kosoko AM, Olurinde OJ, Akinloye AY. Doxorubicin induced neuro- and cardio toxicities in experimental rats: Protection against oxidative damage by Theobroma cacao Stem bark. Biochemistry and Biophysics Reports. 2017;10:303-17.

2. Haybar H, Goudarzi M, Mehrzadi S, Aminzadeh A, Khodayar MJ, Kalantar $\mathrm{M}$, et al. Effect of gemfibrozil on cardiotoxicity induced by doxorubicin in male experimental rats. Biomedicine Pharmacotherap. 2019;109:530-5.

3. Francis A, Nayak Y. Modulation of Doxorubicin-Induced Cardiotoxicity by Averrhoabilimbiextract. J Young Pharm. 2017;9(1):70-8.

4. Bode AM, Zong D. Review- The Two Faces of Capsaicin. Cancer Res. 2011;71(8):2809-14.

5. Basith S, Cui M, Hong S, Choi S. Review- Harnessing the Therapeutic Potential of Capsaicin and Its Analogues in Pain and Other Diseases. Molecules. 2016;966(21):1-28.

\section{PICTORIAL ABSTRACT}

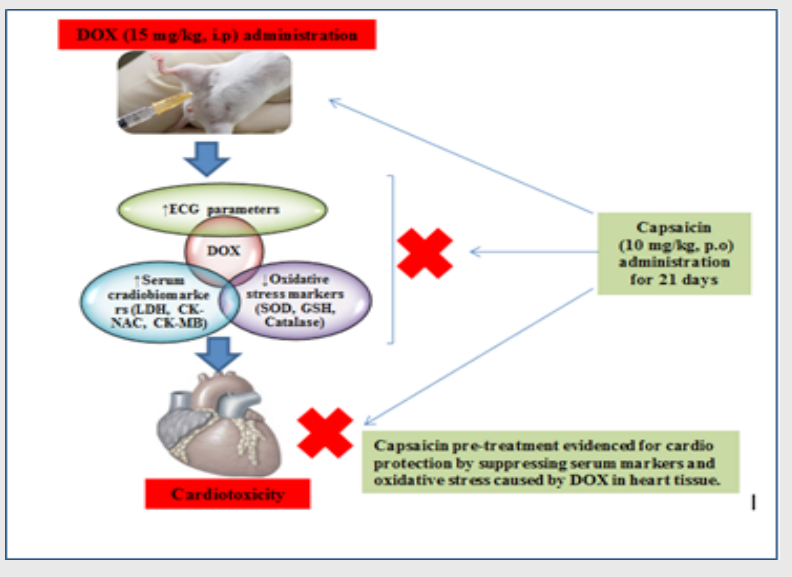

6. Momin FN, Kalai BR, Shikalgar TS, Naikwade NS. Cardioprotective effect of methanolic extract of Ixora coccinea Linn. Leaves on doxorubicin-induced cardiac toxicity in rats. Indian J Pharmacol. 2012;44(2):178-83.

7. Ellmann GL. Tissue sulfhydril groups. Arch Biochem Biophys. 1959;82(1):707.

8. Aebi H. Catalase, in: H.U. Bergmeyer (Ed.), Methods in Enzymatic Analysis, Academic Press, New York. 1983;3:276-86.

9. Kakkar PS, Das B, Viswanathan PNA. Modified spectrophotometric assay of superoxide dismutase. Indian J Biochem Biophys. 1984;21(2):130-2.

10. Bancroft JD, Gamble M. Theory and Practice of Histological Techniques, $5^{\text {th }}$ ed., Churchill Livingstone Pub. Edinburgh/New York/London/Philadelphia. 2002;125-38.

11. Singh S, Dhasmana DC, Bisht M, Singh PK. Pattern of adverse drug reactions to anticancer drugs: a quantitative and qualitative analysis. Indian J Med Paediatr Oncol. 2017;38(2):140- 5.

12. El-Agamy DS, El-Harbi KM, Khoshhal S, Ahmed N, Elkablawy MA, Shaaban $\mathrm{AA}$, et al. Pristimerin protects against doxorubicin-induced cardiotoxicity and fibrosis through modulation of Nrf2 and MAPK/NF-kB signalling pathways. Cancer Management Res. 2019;11:47-61.

13. Kwatra M, Kumar V, Jangra A, Mishra M, Ahmed S, Ghosh P, et al. Ameliorative effect of naringin against doxorubicin-induced acute cardiac toxicity in rats. Pharm Biol. 2016;54(4):637-47.

14. Rollyson WD, Stover CA, Brown KC, Perry HE, Stevenson CD, McNees CA. et al. Bioavailability of capsaicin and its implications for drug delivery. J Control Release. 2014;196:96-105.

15. Sun F, Xiong S, Zhu Z. Review- Dietary Capsaicin Protects Cardiometabolic Organs from Dysfunction. Nutrients. 2016;174(8):1-13.

16. Sayadi O, Puppala D, Ishaque N, Doddamani R, Merchant FM, Barrett C, et al. A novel method to capture the onset of dynamic electrocardiographic ischemic changes and its implications to arrhythmia susceptibility. J Am Heart Assoc. 2014;3(5):e001055.

17. Singh MK, Mohd F, Ayaz A, Ankur S, Jyoti Y. Protective effect of Lagenaria siceraria against doxorubicin induced cardiotoxicity in wistar rats. Int J Drug Dev Res. 2012;4(2):298-305.

18. Qin SL, Liu SL, Wang RR. Protective effect of capsaicin against myocardial ischemia reperfusion injury of rat in vivo. Sichuan Da Xue Xue Bao Yi Xue Ban. 2008;39(4):550-4.

19. Okabe E, Odajima C, Taga R, Kukreja RC, Hess ML, Ito H. The effect of oxygen free radicals on calcium permeability and calcium loading at steady state in cardiac sarcoplasmic reticulum. Mol Pharmacol. 1988;34(3):388-94.

20. Aydin B. The effects of capsaicin and vitamin $E$ on high fat diet induced obesity, hyperlipidemia and oxidative stress in different organs of mice. J Food Nutr Res. 2015;3(6):357-64.

\section{SUMMARY}

The current study illustrated the cardioprotective role of CAP against DOX induced cardiotoxicity in wistar rats. In our study, CAP significantly ameliorates DOX induced cardiac damage mainly by regulating ECG parameters, suppressing the increased serum markers and potentiate the depleted antioxidant parameters caused by DOX.

Cite this article: Karale S, Yamuna PV, Kamath JV. Protective Effect of Capsaicin against Doxorubicin Induced Cardiotoxicity in Experimental Rats. Indian J of Pharmaceutical Education and Research. 2020;54(1):95-100. 